\title{
Société Canadienne d'Études de la Renaissance, Statuts et Reglements
}

\section{Article I: NOM}

La société a pour nom Société canadienne d'études de la Renaissance/ Canadian Society for Renaissance Studies.

Article II: BUTS

La Société canadienne d'études de la Renaissance a pour buts de promouvoir et développer au Canada les études sur la Renaissance, sur une base multidisciplinaire; et de diffuser l'information concernant les recherches en cours, les rencontres et les publications, tant canadiennes qu'internationales.

\section{Article III: LES MEMBRES}

Peuvent devenir membres de la société

1. Les personnes engagées dans l'enseignement et la recherche dans le domaine de la Renaissance, y compris les candidates à la maîtrise et au doctorat.

2. Toute personne souscrivant aux buts de la Société.

\section{Article IV: L'ASSEMBLEE GENERALE}

1. La Société sera tenue de se réunir en assemblée générale une fois par an.

2. L'assemblée générale est convoquée soit par décision du Bureau soit la demande de $25 \%$ au moins des membres; mais toute réunion de l'assemblée générale doit être précédée d'un avis donné au moins six semaines d'avance à tous les membres.

\section{Article V: LE BUREAU}

Le bureau de la Société aura la responsabilité de gérer ses affiares, de convoquer l'assemblée générale et de mettre à exécution les décisions de celle-ci. 
84 / Renaissance and Reformation

Il sera composé de: - Un président

- un vice-président

- un secrétaire-trésorier

Il y aura en outre sept membres assesseurs représentant aussi équitablemen que possible les disciplines contribuant à l'étude de la Renaissance ainsi que les différentes régions du Canada. Les membres du bureau sont nommé: pour deux ans.

\section{Article VI: AMENDEMENTS}

Les présents Statuts pourront être amendés soit par deux tiers de: membres présents et votant en Assemblée générale à condition qu'un avi écrit d'amendement ait été donné au moins six semaines d'avance l'ensemble des membres, soit par la majorité de deux tiers des membre votant lors d'un scrutin tenu par la poste, à condition que dans ce ca l'amendement ait été proposé par le bureau ou par cinq membres de li Société et qu'un avis écrit d'amendement ait été donné au moins sir semaines d'avance à l'ensemble des membres. 